\title{
Learning Aggregate Functions with Neural Networks Using a Cascade-Correlation Approach
}

\author{
Werner Uwents ${ }^{1}$ and Hendrik Blockeel ${ }^{1,2}$ \\ ${ }^{1}$ Department of Computer Science, Katholieke Universiteit Leuven \\ ${ }^{2}$ Leiden Institute of Advanced Computer Science, Leiden University
}

\begin{abstract}
In various application domains, data can be represented as bags of vectors. Learning functions over such bags is a challenging problem. In this paper, a neural network approach, based on cascade-correlation networks, is proposed to handle this kind of data. By defining special aggregation units that are integrated in the network, a general framework to learn functions over bags is obtained. Results on both artificially created and real-world data sets are reported.
\end{abstract}

\section{Introduction and Context}

In general, two types of approaches to relational data mining can be distinguished. In the first type, the propositionalization approach, each data element is summarized into a vector of fixed length. We will refer to the components of this vector as features. In the second type, the direct approach, no summarization is performed and structured data elements are handled directly. A search is performed through a large hypothesis space, which may contain for instance (sets of) logical clauses.

The hypotheses searched by a relational learner could themselves be considered features of the data, i.e., the search for a suitable hypothesis can be seen as a search in a feature space. For instance, in the context of ILP, typically the final model built is a set of clauses, where the clauses are learned one by one. The single clauses could be said to be boolean features, combined into a disjunction.

From this point of view, propositionalization approaches are in principle equally powerful as direct approaches. In practice, including a separate feature for each clause in the search space in the fixed-size vector is often not feasible because the feature space is too large and might even be infinite. Direct approaches can be seen as "lazy propositionalization" approaches: they perform a greedy search through the feature space, gradually constructing relevant features.

If we look at the kind of features that are constructed by a relational learner, an essential property of these features is that they map sets of objects to a single scalar value. Such functions are called aggregate functions and they play a key role in relational learners. For instance, in ILP, if we have a clause happy f ather (X) :$\operatorname{child}(\mathrm{Y}, \mathrm{X})$, the "feature" constructed is essentially of the form $\exists y: \operatorname{Child}(y, x)$, which tests if the set of all $y$ 's related to $x$ through the Child relation is empty or not. 
As has been pointed out by Blockeel and Bruynooghe [1, the features constructed by relational learning systems are generally of the form $\mathcal{F}\left(\sigma_{C}(S)\right)$ with $S$ a set of objects, $C$ a condition defined over single objects, $\sigma$ the selection operator from relational algebra, and $\mathcal{F}$ an aggregate function, which maps a set to a scalar. In the above clause, $S$ is the set of children of $x, C$ is true, and $\mathcal{F}$ is the "there exists" operator $(\exists)$. For the clause happy_father (X) :- $\operatorname{child}(\mathrm{Y}, \mathrm{X})$, age (Y,A), A $<12, S$ and $\mathcal{F}$ are the same, but $C$ is now a condition on the age of the children.

Blockeel and Bruynooghe further pointed out that ILP systems typically construct structurally complex conditions but always use the same, trivial, aggregate function, namely $\exists$. The importance of using more complex aggregate functions has been recognized by many people [2]3. Most systems that handle such complex aggregate functions follow the propositionalization approach. The reason for this is that the structure of the search space of features of the form $\mathcal{F}\left(\sigma_{C}(S)\right)$ becomes much more complex and difficult to search when $\mathcal{F}$ can be something else than $\exists[4$. But to keep the propositionalization approach feasible, a limited set of $\mathcal{F}$ functions still needs to be used, and the number of different $C$ considered for $\sigma_{C}$ must remain limited. For instance, Krogel and Wrobel [3] allow a single attribute test in $C$, but no conjunctions.

Given the limitations of the propositionalization approach, it is useful to study how direct approaches could include aggregate functions other than $\exists$. More recently, methods for learning relevant features of the form $\mathcal{F}\left(\sigma_{C}(S)\right.$ ) have been proposed. Vens, Van Assche et al. [5]6] proposed a random forest approach that avoids the problems of searching a complex-structured search space, while Vens [4] studied the monotonicity properties of features of the form $\mathcal{F}\left(\sigma_{C}(S)\right)$ and showed how efficient refinement of such features is possible for the most commonly occurring aggregate functions.

In parallel, Uwents et al. studied to what extent subsymbolic concepts on relational data can be learned using neural network approaches. Recurrent neural networks were first proposed to learn the aggregate features, leading to the concept of relational neural networks [7]. While a regular network maps one input vector to an output vector, recurrent networks can map a sequence of input vectors to a single output vector. This property was exploited to handle sets of vectors, the elements of which were input in random order in the network.

From the explanation above, it is clear that learning aggregate features from sets is a crucial part of any relation learner. In this paper, we therefore focus on the subsymbolic learning of aggregate functions as such, without considering the possibility of having many different relationships. This means that the general relational learning setting is restricted to the situation where there is just a single one-to-many relationship. This relationship results in bags of vectors with associated target vectors. This resembles the multi-instance setting, because in multi-instance learning, one also deals with data sets containing a bag of vectors for each data instance. However, in multi-instance learning the hypothesis that should be learned has some restrictions. Each bag is classified as positive or negative but this classification can be reformulated in terms of a classification 
of the individual vectors in the bag. If all vectors are negative, the bag as a whole will also be classified as negative. If there is at least one positive vector, the bag will be classified as positive. Learning aggregate functions in general is more complicated because these restrictions do no longer apply and the class of possible aggregate functions is very broad and diverse. In this paper, we will consider the use of neural networks for learning this kind of general aggregate functions. For the multi-instance setting, the use of neural networks has been explored before by Ramon and De Raedt [9]. They make use of a softmax function in the network, similar to what will be used in our aggregate cascade-correlation networks as explained in section 2.2 .

The remainder of the paper is structured as follows. In section 2 we review cascade-correlation networks and we adapt these networks so that they can represent aggregation functions, this by introducing a limited number of aggregation units in them. A training procedure for such networks is also described. In section 3 we experimentally evaluate the method, to conclude in section 4 .

\section{Aggregate Cascade-Correlation Networks}

Cascade-correlation networks are a special kind of neural networks, constructed one unit at a time. In the next subsection, the original cascade-correlation algorithm will be discussed. After that, a number of new units, capable of aggregating, will be presented. These units will then be integrated in an adapted version of cascade-correlation, resulting in a network structure that can learn concepts with aggregation. The resulting networks are called aggregate cascadecorrelation networks (ACCNs).

\subsection{The Original Cascade-Correlation Network}

The idea behind the original cascade-correlation algorithm [10] is to learn not only the weights, but also the structure of the network at the same time. This is done in a constructive way, meaning that only one neuron at a time is trained and then added to the network. One starts with a network without any hidden unit, and then hidden neurons are added, one by one, until some stopping criterion is satisfied. Once a hidden neuron has been added to the network, its weights remain fixed throughout the rest of the procedure. This also means that, besides the actual input vector, the output values of these existing hidden units can be used as extra inputs for any new hidden neuron. At the output, a linear function can be used. A schema of the network is shown in figure 1 For an input vector $x_{p}$, the output values $o_{p, k}$ of the network are then computed as

$$
\begin{aligned}
o_{k}\left(x_{p}\right) & =\sum_{i=1}^{N} v_{k, i} x_{p, i}+\sum_{i=1}^{H} v_{k, i} h_{i}\left(x_{p}\right)+v_{k, N+H+1} \\
h_{i}\left(x_{p}\right) & =\sigma\left(\xi_{i}\left(x_{p}\right)\right)
\end{aligned}
$$




$$
\begin{aligned}
\xi_{i}\left(x_{p}\right) & =\sum_{j=1}^{N+i} w_{i, j} \theta_{i, j}\left(x_{p}\right) \\
\theta_{i}\left(x_{p}\right) & =\left[x_{p, 1}, \ldots, x_{p, N}, h_{1}\left(x_{p}\right), \ldots, h_{i-1}\left(x_{p}\right), 1\right]
\end{aligned}
$$

with $N$ the number of inputs and $H$ the number of hidden units, while $w_{i, j}$ and $v_{k, i}$ are the weights for the hidden and the output units.

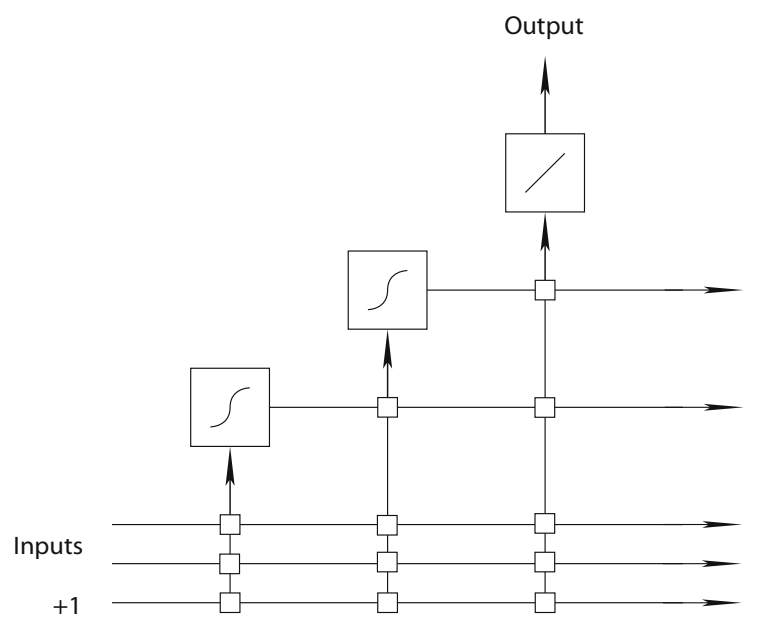

Fig. 1. Schema of the feedforward cascade-correlation network

The training of the network is done in two alternating phases. Before a new hidden neuron is added, its weights are trained while keeping the weights of all other hidden units fixed. This training is not done by minimizing the squared error between target and output, but by maximizing the correlation with the residual error. The residual error is defined as the difference between the actual target value and the output of the existing network, before adding the new neuron. Instead of the real correlation, a slightly different measure $S$ is taken, in which some normalization factors are omitted and the absolute value is taken:

$$
\begin{aligned}
S & =\sum_{k=1}^{K}\left|s_{k}\right| \\
s_{k} & =\sum_{p=1}^{P}\left(h_{H+1}\left(x_{p}\right)-\overline{h_{H+1}\left(x_{p}\right)}\right)\left(e_{k}\left(x_{p}\right)-\overline{e_{k}\left(x_{p}\right)}\right) \\
e_{k}\left(x_{p}\right) & =o_{k}\left(x_{p}\right)-t_{p, k}
\end{aligned}
$$

with $K$ the number of outputs, $P$ the number of patterns in the training data, $h_{H+1}$ the new candidate unit and $t_{p}$ the target vector for pattern $p$. When this $S$ value is maximized, the output of the new hidden neuron will correlate well with 
the residual error. The key idea here is that a unit that correlates well with the residual error, will help to reduce the output error when added to the network. The maximization is done by computing the gradient and performing some form of gradient descent. This gradient is computed as follows:

$$
\frac{\partial S}{\partial w_{i}}=\sum_{k=1}^{K} \sum_{p=1}^{P} \operatorname{sign}\left(s_{k}\right) \sigma^{\prime}\left(\xi_{H+1}\left(x_{p}\right)\right)\left(e_{k}\left(x_{p}\right)-\overline{e_{k}\left(x_{p}\right)}\right) \theta_{H+1, i}\left(x_{p}\right)
$$

Instead of training only one candidate neuron at a time, a pool of neurons, initialized with random weights, can be trained. At the end, the best one is selected. This increases the chance that a good candidate will be found. Once the best candidate is selected and added to the network, the output weights for the updated network can be trained. If a linear function is used at the outputs, the output weights can be obtained by simple linear regression.

\subsection{Cascade-Correlation with Aggregation Units}

The concept of cascade-correlation networks can be extended to networks for learning aggregate functions. The crucial difference is that instead of the simple hidden neurons, units that can process bags are used. For the rest, the network and the training of it works in the same way as for the feedforward networks. The data set now consists of bags $b_{p}=\left\{x_{p, 1}, \ldots, x_{p, m}\right\}$, where $x_{p, i}$ are vectors of size $N$, with associated target vectors $t_{p}$. Because the input is a bag of vectors instead of one single vector, it can no longer be used as direct input for the output units, and so this is dropped from the equation. Each input bag $b_{p}=\left\{x_{p, 1}, \ldots, x_{p, m}\right\}$ of a pattern $p$ will be processed by the hidden units. Each time a vector of the bags has been processed, an intermediate output value for the hidden units can be computed, yielding a sequence of $m$ values for each hidden unit. The final value is used by the output units, but the whole sequence of values can also be used by new hidden units. A schema of an aggregate cascade-correlation network for 2 input vectors is shown in figure 2, The network then becomes:

$$
\begin{aligned}
o_{k}\left(b_{p}\right) & =\sum_{i=1}^{H} v_{k, i} h_{i, m}\left(b_{p}\right)+v_{k, H+1} \\
h_{i, j}\left(b_{p}\right) & =g_{i}\left(\left\{\xi_{i}\left(x_{p, 1}\right), \ldots, \xi_{i}\left(x_{p, j}\right)\right\}\right) \\
\xi_{i}\left(x_{p, j}\right) & =\sum_{l=1}^{N+i} w_{i, l} \theta_{i, l}\left(x_{p, j}\right) \\
\theta_{i}\left(x_{p, j}\right) & =\left[x_{p, 1}, \ldots, x_{p, N}, h_{1, j}\left(x_{p}\right), \ldots, h_{i-1, j}\left(x_{p}\right), 1\right]
\end{aligned}
$$

with $g_{i}$ a special function. Different functions can be used for $g_{i}$, as long as they perform some kind of aggregation. One could take the sum or the max function for instance. Another possibility is to use a locally recurrent neuron. The only condition imposed on the $g_{i}$ function is that it must be derivable to allow gradient training. Different types of units can easily be combined in the 


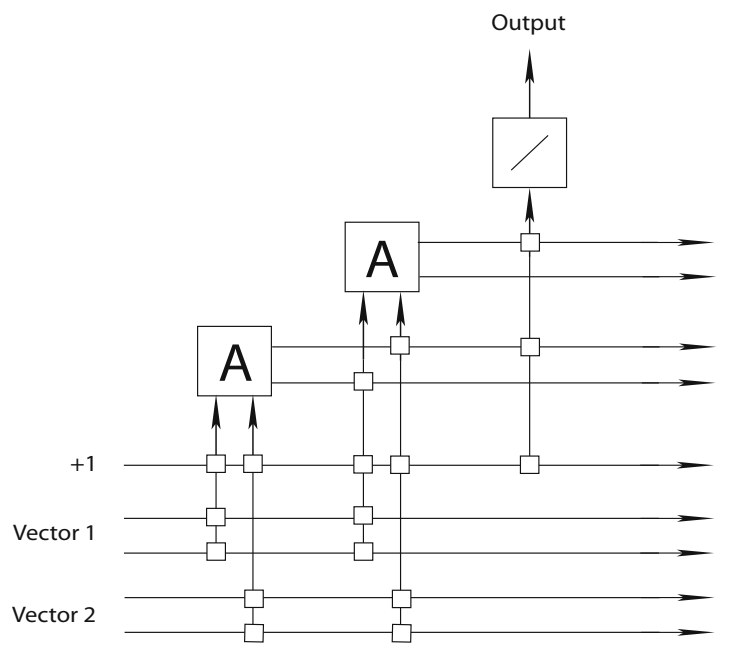

Fig. 2. Schema for an aggregate cascade-correlation network with 2 input vectors

network. For every new unit, several candidates of different types can be trained and the best one selected and added to the network. The four different types that will be considered here, are explained below.

1. The simplest type of unit is probably the sum unit:

$$
\begin{aligned}
g_{i}^{\text {sum }}\left(b_{p}\right) & =\sum_{j=1}^{m} \sigma\left(\xi_{i}\left(x_{p, j}\right)\right) \\
\frac{\partial}{\partial b_{p}} g_{i}^{\text {sum }}\left(b_{p}\right) & =\sum_{j=1}^{m} \sigma^{\prime}\left(\xi_{i}\left(x_{p, j}\right)\right)
\end{aligned}
$$

2. Somewhat more complicated is the max unit because the maximum function is not easily derivable. To circumvent this, one could use the softmax function. This is the same approach as in [9]. The $\operatorname{smx}$ unit is then defined as:

$$
\begin{aligned}
g_{i}^{s m x}\left(b_{p}\right) & =\frac{1}{C} \log \left(\sum_{j=1}^{m} e^{C \cdot \sigma\left(\xi_{i}\left(x_{p, j}\right)\right)}\right) \\
\frac{\partial}{\partial b_{p}} g_{i}^{s m x}\left(b_{p}\right) & =\sum_{j=1}^{m} \frac{e^{C \cdot \sigma\left(\xi_{i}\left(x_{p, j}\right)\right)}}{\sum_{l=1}^{m} e^{C \cdot \sigma\left(\xi_{i}\left(x_{p, l}\right)\right)}} \sigma^{\prime}\left(\xi_{i}\left(x_{p, j}\right)\right)
\end{aligned}
$$

$C$ is a constant and the larger the value of $C$, the closer this function will approximate the real max function. 
3. Instead of choosing a value for $C$ that is large enough to give a good approximation, one could take the limit for $C$ going to infinity, which gives the real max function and its derivative:

$$
\begin{aligned}
\max \left\{x_{1}, \ldots, x_{n}\right\} & =\lim _{C \rightarrow \infty} \frac{1}{C} \sum_{i=1}^{n} e^{C \cdot x_{i}} \\
\frac{\partial}{\partial x_{j}} \max \left\{x_{1}, \ldots, x_{n}\right\} & = \begin{cases}1 & \text { if } x_{j}=\max \left\{x_{1}, \ldots, x_{n}\right\} \\
0 & \text { otherwise }\end{cases}
\end{aligned}
$$

A possible disadvantage with this exact function is that the derivative is not continuous anymore, which can deteriorate learning. The corresponding max unit then becomes:

$$
\begin{aligned}
g_{i}^{\max }\left(b_{p}\right) & =\max \left(\left\{\sigma\left(\xi_{i}\left(x_{p, 1}\right)\right), \ldots, \sigma\left(\xi_{i}\left(x_{p, k}\right)\right)\right\}\right) \\
\frac{\partial}{\partial b_{p}} g_{i}^{s m x}\left(b_{p}\right) & =\sigma^{\prime}\left(\xi_{i}\left(x_{p, l^{*}}\right)\right) \quad \text { with } \sigma\left(\xi_{i}\left(x_{p, l^{*}}\right)\right)=g_{i}^{\max }\left(b_{p}\right)
\end{aligned}
$$

4. The fourth type of unit considered here, is the locally recurrent unit. This is a standard feedforward unit with one recurrent connection with itself added. This $l r c$ unit is defined as:

$$
g_{i}^{l r c}\left(b_{p}\right)=\sigma\left(\xi_{i}\left(x_{p, k}\right)+w_{r} g_{i}^{l r c}\left(b_{p} \backslash\left\{x_{p, k}\right\}\right)\right)
$$

Gradient computation for this type of unit is done using backpropagation through time [1].

New types of units could easily be invented if necessary, but only these four will be considered in the rest of the paper.

\subsection{Aggregate Cascade-Correlation Training}

With all parts of the aggregate cascade-correlation network explained, it only remains to discuss the training of the network in more detail. Each time a new unit should be added to the hidden layer, a pool of units is created of the four types discussed in the previous subsection. Weights are initialized randomly. After that, all units in the pool are trained for a number of iterations, similar to backpropagation. This training is basically a gradient ascent, maximizing the correlation with the outputs as given in formula 5 . The computation of the gradient depends of course on the type of unit. The gradient ascent itself is actually done by using resilient propagation, as described in [12. This method has the advantage that the step size is determined automatically and convergence is faster than for a fixed step size. The basic idea is to increase the step size when the sign of the gradient remains the same, and decrease the step size when the sign changes.

When all units in the pool have been trained, the best one is chosen. In this case, the best unit is the one with the highest correlation. To be able to 
compare units of different types with each other, the absolute value of the real correlation has to be computed, and not the $S$-value from formula 5 in which some normalization constants were omitted. When the unit with the highest correlation has been chosen, it is installed in the network and the output weights have to be learned again. Because linear activation functions are used for the output units, the output weights can be determined with least squares linear regression.

In the ideal case, when there is enough data available, a validation set can be used to determine when to stop adding new units. If this is difficult, there is also an alternative stopping criterion. Typically, the first units added to the network will have a high correlation. When more units are added, the correlation will decrease until no more reduction can be made. One can stop training when the correlation is below a certain threshold or does not decrease significantly anymore.

\section{Experiments}

In this section, a number of experimental results will be discussed. First, a series of experiments is carried out on artificially created data sets. After that, the method is evaluated on some real-world data sets.

\subsection{Simple Aggregates}

A simple experiment to examine the capacity of the aggregate cascade-correlation network, is to create artificial data with predefined aggregate functions and train the networks on it. The data consists of bags with a variable number of elements. Each element of the bag is a vector with five components. Only the first or the first and second component are relevant for the target value, depending on the aggregate function under consideration. The values of these components are randomly generated, but in such a way that the target values are uniformly distributed over the possible target values. All the other components are filled with uniformly distributed random numbers from the interval $[-1,1]$. It is very likely that he number of vectors in the bags influences the difficulty of the learning task, so different sizes are tested. The data sets denoted as small contain 5 to 10 vectors per bag, the medium data sets 20 to 30 and the large ones 50 to 100. Each data set contains 3000 bags. A range of different aggregate functions are used to construct the data sets:

1. count: the target is the number of vectors in the bag.

2. sum: the target is the sum of all values of the first component of the bag vectors.

3. max: the target is the maximum value of the first component of the bag vectors.

4. avg: the target is the average value of the first component of the bag vectors.

5. stddev: the target is the standard deviation of the values of the first component of the bag vectors. 
6. cmpcount: the target is the number of bag vectors for which the value of the first component is smaller than the value of the second component.

7. corr: the target is the correlation between the first two components of the bag vectors.

8. even: the target is one if the number of positive values for the first component is even, and zero if it is odd.

9. distr: the target is one if the values of the first component come from a Gaussian distribution, and zero if they are from a uniform distribution.

10. select: the target is one if at least one of the values of the first component lies in a given interval, and zero otherwise.

11. conj: the target is one if there is at least one vector in the bag for which the the first and the second component lie in a certain interval.

12. disj: the target is one if there is at least one vector in the bag for which the first or the second component lies in a certain interval.

The first 7 data sets have a numerical target, the others a nominal target. In case of a nominal target, the number of positive and negative examples are equal. The experiments are done using 10-fold cross-validation. One fold is used as test set, 7 folds are used for training and 2 folds are used as validation set to determine when to stop adding units. The maximum number of hidden units is limited to 10 . The number of candidate units trained in every step is 20 , which means that there are five units of every type. Each unit is trained for 500 iterations, which should be more than enough to have converged to optimal weights. For the data sets with nominal target, the accuracy is reported and for the sets with numerical targets the mean squared error is given. Standard deviation is reported as well. The results are summarized in table 1

From the results, it is clear that most functions can be learned very well. Only the even function is really impossible to learn it seems. For the distr function, the number of vectors must be large to be able to learn it well. This makes sense because it is easier to say whether a bag of values comes from a normal or uniform distribution if the bag is larger than when it is rather small. In table2, results are given using fully recurrent networks, similar to what is used in relational neural networks [7]. Compared with these results, it is clear that ACCNs perform better. One of the major problems with these recurrent networks, is the decreasing performance on larger bags. If we look at the results for the select data sets for instance, then the accuracy on the data set with small bags is still reasonable for the recurrent network, although the accuracy for the ACCNs is better. But for the data sets with larger bags, the accuracy goes down for the recurrent networks while it remains about the same for the ACCNs. Overall, it is clear that ACCNs are a better choice than the recurrent networks.

\section{$3.2 \quad$ Trains}

The trains data sets are also artificially created data sets containing a number of trains. Every train consists of a number of cars, carrying some load. Some of the trains are eastbound, the others are westbound. The direction of the trains 
Table 1. Results for the simple aggregate data sets with aggregate cascade-correlation networks. Accuracies or mean squared errors are given, depending on whether the target is numeric or nominal, together with the standard deviations. The columns small, medium and large refer to the size of the bags in the data sets.

\begin{tabular}{|c|c|c|c|c|}
\hline & & small & medium & large \\
\hline & count & $0.00(0.00)$ & $0.00(0.00)$ & $0.00(0.00)$ \\
\hline & sum & $0.00(0.00)$ & $0.00(0.00)$ & $0.00(0.00)$ \\
\hline & $\max$ & $0.00(0.00)$ & $0.00(0.00)$ & $0.00(0.00)$ \\
\hline & $\operatorname{avg}$ & $0.04(0.03)$ & $0.01(0.01)$ & $0.01(0.01)$ \\
\hline & stddev & $0.02(0.02)$ & $0.01(0.01)$ & $0.00(0.00)$ \\
\hline & cmpcount & $0.00(0.00)$ & $0.00(0.00)$ & $0.00(0.00)$ \\
\hline & corr & $0.04(0.03)$ & $0.02(0.02)$ & $0.01(0.01)$ \\
\hline \multirow{5}{*}{ 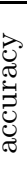 } & even & $51.59(0.05)$ & $51.10(0.04)$ & $51.34(0.10)$ \\
\hline & distr & $66.48(0.05)$ & $77.97(0.07)$ & $84.23(0.09)$ \\
\hline & select & $99.21(0.07)$ & $99.13(0.10)$ & $98.83(0.10)$ \\
\hline & conj & $100.00(0.04)$ & $100.00(0.00)$ & $99.89(0.10)$ \\
\hline & disj & $99.13(0.06)$ & $98.57(0.10)$ & $96.54(0.11)$ \\
\hline
\end{tabular}

Table 2. Results for the simple aggregate data sets with fully recurrent networks. Accuracies or mean squared errors are given, depending on whether the target is numeric or nominal, together with the standard deviations. The columns small, medium and large refer to the size of the bags in the data sets.

\begin{tabular}{|c|c|c|c|c|}
\hline & & small & medium & large \\
\hline \multirow{7}{*}{ II } & count & $0.06(0.10)$ & $0.26(0.01)$ & $0.23(0.01)$ \\
\hline & sum & $0.00(0.00)$ & $0.09(0.10)$ & $0.23(0.01)$ \\
\hline & $\max$ & $0.02(0.00)$ & $0.01(0.00)$ & $0.01(0.01)$ \\
\hline & avg & $0.02(0.00)$ & $0.01(0.01)$ & $0.01(0.01)$ \\
\hline & stddev & $0.03(0.00)$ & $0.04(0.00)$ & $0.04(0.01)$ \\
\hline & cmpcount & $0.02(0.01)$ & $0.01(0.01)$ & $0.11(0.07)$ \\
\hline & corr & $0.07(0.01)$ & $0.08(0.04)$ & $0.04(0.01)$ \\
\hline & even & $52.64(0.01)$ & $50.19(0.02)$ & $50.23(0.01)$ \\
\hline 8 & distr & $51.62(0.05)$ & $63.49(0.02)$ & $58.70(0.02)$ \\
\hline$\exists$ & select & $90.55(0.03)$ & $63.47(0.03)$ & $50.42(0.02)$ \\
\hline 8 & conj & $94.43(0.01)$ & $68.09(0.10)$ & $49.11(0.05)$ \\
\hline & disj & $72.08(0.03)$ & $51.24(0.05)$ & $50.32(0.02)$ \\
\hline
\end{tabular}

is what has to be learned and this target concept is based on the properties of the cars of a train and their loads. The cars of the train constitute a bag for each train. A data generator [13] for this train problem was used to create 12 data sets with different properties. Sets 1 to 4 consist of short trains, having 2 to 6 cars. Data sets 5 to 8 are similar to sets 1 to 4 , except that they contain longer trains. Each of these trains consists of 20 to 29 cars. The used concepts are the same as for sets 1 to 4, except that the numbers in the aggregations are adapted to the longer length of the trains. Data sets 9 to 12 contain noisy data. 
This means that a number of samples have been mislabeled. The used concepts for the different data sets are as follows:

1. Trains having at least one circle load are eastbound, the others are westbound.

2. Trains having at least one circle or rectangle load and at least one car with peaked roof or 3 wheels are eastbound, the others are westbound.

3. Trains having more than 8 wheels in total are eastbound, the others are westbound.

4. Trains having more than 3 wheels in total and at least 2 rectangle loads and maximum 5 cars are eastbound, the others are westbound.

5. Same concept as for set 1 .

6. Same concept as for set 2 .

7. Trains having more than 53 wheels in total are eastbound, the others are westbound.

8. Trains having more than 45 wheels in total and at least 10 rectangle loads and maximum 27 cars are eastbound, the others are westbound.

9. Same concept as for set 1 , but with $5 \%$ noise.

10. Same concept as for set 1 , but with $15 \%$ noise.

11. Same concept as for set 3, but with $5 \%$ noise.

12. Same concept as for set 3 , but with $15 \%$ noise.

The training setup is the same as for the simple aggregate data sets. The results for ACCNs and fully recurrent networks are given in table 3, It is clear that most concepts can be learned well with ACCNs. Most of the data sets without noise have an accuracy very close to $100 \%$. Only for set 8 , which has the most difficult concept, is it impossible to get close to perfect accuracy. For the data sets with noise, the accuracies are all close to $100 \%$ minus the percentage of noise, which means that the method is noise-resistant. Compared with the fully recurrent networks, the results for ACCNs are always better again. Sometimes the difference is quite spectacular. Also for these data sets, the size of the bags has an important influence on the accuracy for the recurrent networks.

\subsection{Musk}

Musk is a well-known multi-instance data set [14]. Each data instance stands for a molecule, represented by a bag of all its possible conformations. A conformation is described by 166 numerical features. The molecules have to be classified as musk or non-musk. The data set consists of two parts. The first part contains 92 molecules, the second part 102. In each bag, there are between 2 and 40 conformations for the first part, and between 1 and 1044 for the second part.

Experiments were carried out using 10-fold cross-validation. For the ACCNs, a pool of 20 neurons and 500 training iterations are used in every step. The value of the correlation is used as stopping criterion as described above. The results for the musk data sets can be found in table 4. The accuracies for ACCNs are not bad but not that excellent either compared with the other methods. Some 
Table 3. Average accuracy and standard deviation using 10-fold cross-validation for the trains data sets. ACCNs stands for aggregate cascade-correlation networks and FRNs for fully recurrent networks.

\begin{tabular}{|l|c|c|}
\hline & ACCNs & FRNs \\
\hline \hline 1 & $99.8(0.2)$ & $99.7(0.1)$ \\
2 & $100.0(0.0)$ & $97.1(0.2)$ \\
3 & $100.0(0.0)$ & $99.9(0.1)$ \\
4 & $98.7(0.4)$ & $92.7(0.3)$ \\
5 & $99.7(0.6)$ & $92.5(0.2)$ \\
6 & $99.4(0.5)$ & $73.9(0.3)$ \\
7 & $99.3(0.6)$ & $51.3(0.5)$ \\
8 & $84.6(8.4)$ & $71.0(0.3)$ \\
9 & $95.8(0.3)$ & $93.6(0.2)$ \\
10 & $85.7(0.1)$ & $81.7(0.4)$ \\
11 & $95.6(0.2)$ & $93.0(0.3)$ \\
12 & $85.3(0.2)$ & $82.0(0.3)$ \\
\hline
\end{tabular}

Table 4. Accuracies and 95\% confidence intervals for the musk data sets using 10 -fold crossvalidation. Results for other methods than ACCN are obtained from 14.

\begin{tabular}{|l|c|c|}
\hline & musk 1 & musk 2 \\
\hline \hline iterated discrim APR & $92.4[87.0-97.8]$ & $89.2[83.2-95.2]$ \\
GFS elim-kde APR & $91.3[85.5-97.1]$ & $80.4[72.7-88.1]$ \\
GFS elim-count APR & $90.2[84.2-96.3]$ & $75.5[67.1-83.8]$ \\
GFS all-positive APR & $83.7[76.2-91.2]$ & $66.7[57.5-75.8]$ \\
all-positive APR & $80.4[72.3-88.5]$ & $72.6[63.9-81.2]$ \\
backpropagation & $75.0[66.2-83.8]$ & $67.7[58.6-76.7]$ \\
C4.5 (pruned) & $68.5[40.9-61.3]$ & $58.8[49.3-68.4]$ \\
\hline ACCN & $85.3[83.1-87.5]$ & $75.5[72.6-78.4]$ \\
\hline
\end{tabular}

of these methods were specifically designed for multi-instance problems while ACCNs are more general. This could make the multi-instance methods better suited for this task. On the other hand, ACCNs might also suffer from poor learning because of relatively few data or skewness in the data. The networks are all very small, in most cases with just one hidden unit. If one looks at the type of unit selected, then this is almost always a max or $\operatorname{smx}$ unit. This is the most logical choice in case of a multi-instance problem.

\subsection{Thioredoxin-Fold Proteins}

In this classification task, proteins have to be classified as belonging to the Thioredoxin-fold family or not [15. It is difficult to do this based on the primary sequence of the proteins, for instance by using hidden Markov models, because there is a low conservation of the primary sequence in this family of proteins. One approach to deal with this problem has been to transform the data into 
bags of vectors. In [15, this transformation is done in three steps. First, the primary sequence motif, which is known to exist in all Thioredoxin-fold proteins, is identified. Around this motif, aligned subsequences are extracted. Finally, these windows are mapped to 8-dimensional numeric properties. For further details about this transformation, see [15. The relevant transformation is referred to as motif-based alignment.

The result of this transformation is a data set containing 193 proteins, each described by a bag of 8-dimensional feature vectors. Of these 193 proteins, 25 are labeled positive and 168 negative. The bags contain 35 to 189 vectors. Two different experiments were carried out, the same as in [15. In the first, simple setting, the data set is divided in three parts, using two of them for training and one for testing. The training setup for the ACCNs is the same as for the musk data sets. The results are given in table 5 .ne can see that the results are worse than for a multi-instance learner. Standard deviations are also quite large, especially for the true positive rate. The skewness of the data set is probably responsible for these problems.

Table 5. Results for 3-fold cross-validation on the Thioredoxin-fold proteins data set. True positive and true negative rates are given, together with the standard deviation over ten runs for ACCNs. MIL denotes a multi-instance learner and this result was reported in 15 .

\begin{tabular}{|l|c|c|}
\hline & TP & TN \\
\hline \hline MIL & 0.74 & 0.88 \\
ACCN & $0.614(0.147)$ & $0.838(0.071)$ \\
\hline
\end{tabular}

Results were also obtained for a second, more difficult experiment carried out in [15], in which 5 of the 25 positive proteins were removed, retaining only the 20 most dissimilar ones. The negative examples are divided in 8 folds, the positive examples in 20 folds of 1 example each. A jack-knife test is performed, taking one of the negative folds and one of the positive folds as test set, the other 19 positive folds and one of the negative folds as training set. This yields a total of 160 experiments. Results for this experiment are shown in table 6. The true positive rate is better now than for MIL, but the true negative rate is worse. Overall, the situation seems to be more or less the same as for the musk data sets where it was also not possible to achieve the same accuracy as multi-instance methods.

\subsection{Financial}

For this experiment, the financial data set of the discovery challenge organized at PKDD'99 and PKDD'00 is used [16. The data set contains 8 different relations in total and the goal is to classify loans as good or bad. There are 234 loans, 203 of which are good and 31 bad. This means that the default accuracy is already $86.8 \%$. Each loan is linked to an account and for every account there is a bag 
Table 6. Results for jack-knife tests on the Thioredoxin-fold proteins data set. True positive and true negative rates are given, together with the standard deviation over all 160 experiments for ACCNs. MIL stands for a multi-instance learner and this result was reported in [15].

\begin{tabular}{|l|c|c|}
\hline & TP & TN \\
\hline \hline MIL & 0.75 & 0.75 \\
ACCN & $0.850(0.035)$ & $0.681(0.028)$ \\
\hline
\end{tabular}

Table 7. Results on the financial data set using 10-fold cross-validation. Results for FORF-LA, DINUS-C, RELAGGS and PROGOL are obtained from [17] and [3].

\begin{tabular}{|l|c|}
\hline & accuracy \\
\hline \hline FORF-LA & $90.8(1.7)$ \\
DINUS-C & $85.1(10.3)$ \\
RELAGGS & $88.0(6.5)$ \\
PROGOL & $86.3(7.1)$ \\
\hline ACCN & $87.2(2.3)$ \\
\hline
\end{tabular}

of transactions. The number of transactions varies between 93 and 594. Only these bags will be used by the ACCNs to learn a classification of the loans, all the other information is ignored because we only work with a single bag for each data instance. It can be hoped however that these transactions contain the most important information to learn the target function. The experiments were done using 10-fold cross-validation. Results are summarized in table 7 The accuracy for the ACCNs is not significantly higher than the default accuracy, but neither is the accuracy of the other methods, except FORF-LA. As said before, these other methods also use more information than only the bags of transactions.

\section{Conclusion}

In this paper, a method for learning functions over bags with neural networks has been proposed. The method is based on cascade-correlation networks, extended with aggregation units that are able to process bags of vectors. This yields a general framework, extendable with new types of units if necessary.

Experimental results on artificial data sets are encouraging and indicate that this method is indeed capable of learning a wide variety of aggregate functions over bags. Compared with recurrent networks on these data sets, ACCNs perform significantly better, especially when the size of the bags is large. Experiments on real-world data sets show that the method performs still reasonably well in the multi-instance setting, although not as well as specific methods for this kind of problems. Some properties of these data sets might make it more difficult to train ACCNs on this data. The result for the financial data set is not that good, but similar to other methods like RELAGGS and FORF. 


\section{References}

1. Blockeel, H., Bruynooghe, M.: Aggregation versus selection bias, and relational neural networks. In: Getoor, L., Jensen, D. (eds.) IJCAI 2003 Workshop on Learning Statistical Models from Relational Data, SRL 2003, Acapulco, Mexico (2003)

2. Knobbe, A., Siebes, A., Marseille, B.: Involving aggregate functions in multirelational search. In: Principles of Data Mining and Knowledge Discovery, Proceedings of the 6th European Conference, pp. 287-298. Springer, Heidelberg (August 2002)

3. Krogel, M.A., Wrobel, S.: Transformation-based learning using multirelational aggregation. In: Rouveirol, C., Sebag, M. (eds.) ILP 2001. LNCS (LNAI), vol. 2157, pp. 142-155. Springer, Heidelberg (2001)

4. Vens, C., Ramon, J., Blockeel, H.: Refining aggregate conditions in relational learning. In: Fürnkranz, J., Scheffer, T., Spiliopoulou, M. (eds.) PKDD 2006. LNCS (LNAI), vol. 4213, pp. 383-394. Springer, Heidelberg (2006)

5. Vens, C., Van Assche, A., Blockeel, H., Dzeroski, S.: First order random forests with complex aggregates. In: Camacho, R., King, R., Srinivasan, A. (eds.) ILP 2004. LNCS (LNAI), vol. 3194, pp. 323-340. Springer, Heidelberg (2004)

6. Van Assche, A., Vens, C., Blockeel, H., Dzeroski, S.: First order random forests: Learning relational classifiers with complex aggregates. Machine Learning 64(1-3), $149-182(2006)$

7. Uwents, W., Blockeel, H.: Classifying relational data with neural networks. In: Kramer, S., Pfahringer, B. (eds.) ILP 2005. LNCS (LNAI), vol. 3625, pp. 384-396. Springer, Heidelberg (2005)

8. Uwents, W., Monfardini, G., Blokeel, H., Scarsello, F., Gori, M.: Two connectionists models for graph processing: An experimental comparison on relational data. In: MLG 2006, Proceedings on the International Workshop on Mining and Learning with Graphs, pp. 211-220 (2006)

9. Ramon, J., De Raedt, L.: Multi instance neural networks. In: Raedt, L.D., Kramer, S. (eds.) Proceedings of the ICML-2000 workshop on attribute-value and relational learning, pp. 53-60 (2000)

10. Fahlman, S.E., Lebiere, C.: The cascade-correlation learning architecture. In: Touretzky, D.S. (ed.) Advances in Neural Information Processing Systems. Denver 1989, vol. 2, pp. 524-532. Morgan Kaufmann, San Mateo (1990)

11. Werbos, P.J.: Back propagation through time: What it does and how to do it. Proceedings of the IEEE 78, 1550-1560 (1990)

12. Riedmiller, M., Braun, H.: A direct adaptive method for faster backpropagation learning: The RPROP algorithm. In: Proc. of the IEEE Intl. Conf. on Neural Networks, San Francisco, CA, pp. 586-591 (1993)

13. Michie, D., Muggleton, S., Page, D., Srinivasan, A.: To the international computing community: A new east-west challenge. Technical report, Oxford University Computing Laboratory, Oxford, UK (1994)

14. Dietterich, T.G., Lathrop, R.H., Lozano-Perez, T.: Solving the multiple instance problem with axis-parallel rectangles. Artificial Intelligence 89(1-2), 31-71 (1997)

15. Wang, C., Scott, S.D., Zhang, J., Tao, Q., Fomenko, D.E., Gladyshev, V.N.: A study in modeling low-conservation protein superfamilies. Technical Report UNLCSE-2004-0003, University of Nebraska (2004)

16. Berka, P.: Guide to the financial data set. In: Siebes, A., Berka, P. (eds.) The ECML/PKDD 2000 Discovery Challenge (2000)

17. Vens, C.: Complex aggregates in relational learning. PhD thesis, Department of Computer Science, KULeuven (2007) 